\title{
SOURCE-CHANNEL MATCHING SPACE-TIME DIVERSITY FOR MULTIMEDIA COMMUNICATIONS
}

\author{
H. Zheng \\ Bell-Labs, Lucent Technologies \\ 791 Holmdel-Keyport Rd. \\ Holmdel, NJ 07733 \\ haitaoz@lucent.com
}

\author{
K. J. R. Liu \\ ECE Department \\ University of Maryland \\ College Park, MD 20742 \\ kjrliu@eng.umd.edu
}

\begin{abstract}
A source-channel matching space-time diversity technique is presented for reliable delivery of layer coded multimedia services through wireless channels. The transmission system is transformed to multiple channel layers associated with different diversity gain. Similarly to the parallel transmission proposed for ADSL[8], we develop a parallel transmission scheme to match the source layers to the channel layers. Power control further alters the error performance of each channel layer to achieve the optimal performance.
\end{abstract}

\section{INTRODUCTION}

Dramatic development in the area of personal communications services allows wireless multimedia services. In the near future, it is expected that millions of mobile uses will be able to access their data and other services such as email, e-news, video telephony, map services, electronic banking while on the move. Realtime multimedia services require high reliability with a low bounded time delay and a reasonably high transmission rate. Wireless channels on the other hand are error-prone, time-varying and bandwidth-limited.

Automatic repeat request(ARQ) based techniques can be applied to reduce the error rate over the airlink to a value similar to that on a typical wireline environment. It allows retransmission of the corrupted data, which also yields additional delay. For real-time applications, the number of retransmission is very limited. On the other hand, the derogatory effects of multipath fading in a wireless communication system can be mitigated by employing antenna diversity. It involves multiple transmit antennas and/or receive antennas to allow multiple signal replica at the receiver, which makes it attractive to use for the transmission over band-limited wireless channels $[1,2,3]$. Recently, space-time diversity has raised great interests $[4,5,6,7]$.

In this paper, we consider the problem of supporting multimedia services over wireless systems using multiple transmit/receive antennas. Multimedia is quite different from general data. A very important feature is that it can be represented in terms of a number of layers, each associated with a distinct data stream. Different layers may have distinctly different tolerances to channel errors and the corresponding data streams should be handled differently by the transmitter. In [8], a parallel transmission scheme was proposed for wireline multimedia services, which transmits layers with higher error performance requirement through subchannels with higher channel gain to noise ratio. In this paper, we show the parallel transmission scheme can be incorporated into space-time diversity to provide efficient wireless multimedia services.

\section{LAYERED SPACE-TIME DIVERSITY}

We consider the layered space-time architecture proposed in $[4,5]$. It utilizes multiple antenna arrays at both transmitter and receiver. The diagonal layered space-time (DBLAST) distributes the coded data across diagonals in space-time domain. This structure leads to theoretical rates which increase linearly with the number of antennas. Th vertical layered space-time(VBLAST) code distributes the coded data in space domain to achieve less complexity compared to DBLAST. By employing interference suppression and interference cancellation, the coded data layers can be separated and decoded independently. This results in a much lower decoding complexity compared to the trellis space-time codes which employ ML decoding, especially for large number of transmit antennas. We consider VBLAST in the paper.

The structure of VBLAST code is shown in Figure 1, 


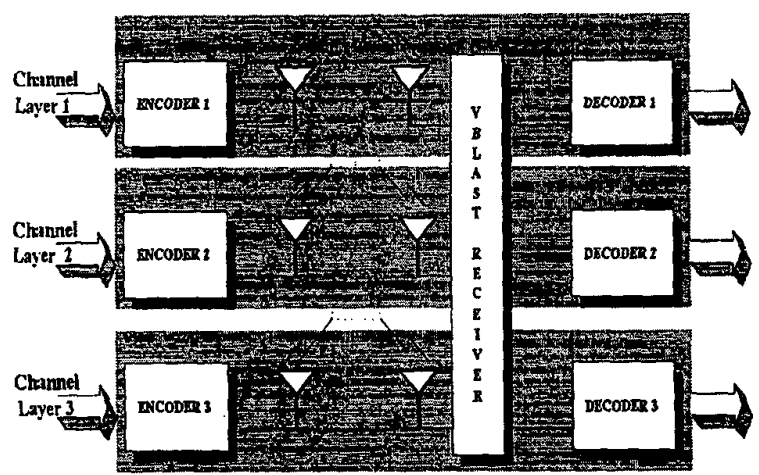

Figure 1: Vertical Layered Space-Time Diversity

assuming $N$ transmit antennas and $M$ receive antennas. Assuming perfect channel estimation at receiver, the decision variables from each horizontal location can be extracted and decoded. The receiver picks one layer, e.g. layer $N-1$, and extracts it by making a decision based on the received vector $\mathbf{r}$. Then the contribution of layer $N-1$ to $r$ is removed. Then, layer $N-2$ can be similarly decoded without the interference from layer $N-1$. Here we refer these layers as channel layers. The average error probability of layer $k$ can be approximated as $[6]$

$\operatorname{Prob}(\mathbf{t} \rightarrow \mathbf{c}) \approx \prod_{\mathbf{c}, \mathbf{t}}\left(\left(|\mathbf{c}-\mathbf{t}|^{2}\right)^{-(M-k)}\right)\left(\frac{E}{N_{0}}\right)^{\sum_{\mathbf{c}, \mathbf{t}, \mathbf{c} \neq \mathbf{t}} M-k}$

By applying Reed Solomon $(N, N-T+1)$ code, the symbol error probability for layer $k$ is then $P e \approx$ $\left(\frac{E d^{\tilde{z}}}{4 N_{\mathrm{c}}}\right)^{-T(M-k)}$, where $d^{2}$ is the minimum distance between two neighboring modulation symbols. Statistically, layer $k$ is associated with diversity gain $M-k$ and should have better error performance than layer $N-1, . . k+1$. Assuming each channel layer uses the same amount of transmitted power, the average SER of the system can be computed as

$$
\begin{aligned}
P e_{a v g}(E) & =\frac{1}{N} \sum_{k=0}^{N-1}\left(\frac{E d^{2}}{4 N_{0}}\right)^{-T(M-k)} \\
& =\frac{1}{N}\left(\frac{E d^{2}}{4 N_{0}}\right)^{-T M} \frac{\left(\frac{E d^{2}}{4 N_{0}}\right)^{T N}-1}{\left(\frac{E d^{2}}{4 N_{0}}\right)^{T}-1},
\end{aligned}
$$

where the average power used $E_{T}=E$. On the other hand, power allocation can also be applied to equalize the error performance of channel layers. That is,

$$
P e_{k}\left(E_{k}\right)=\left(\frac{E_{k} d^{2}}{4 N_{0}}\right)^{-T(M-k)}=\left(\frac{E_{l} d^{2}}{4 N_{0}}\right)^{-T(M-l)}
$$

$$
\rightarrow E_{k}=\left(\frac{E_{0} d^{2}}{4 N_{0}}\right)^{\frac{k}{M-k}} \frac{4 N_{0}}{d^{2}},
$$

where $E_{0}$ represents the transmitted power for channel layer 0. $E_{0}$ is a function of $E_{T}=\frac{1}{N} \sum_{k=0}^{N-1} E_{k}$, and the overall error performance can be represented as a function of $E_{T}$. However, due to the exponential factor $\frac{M}{M-k}$, such allocation leads to huge variation in the transmitted power assigned to the layers. This would yields difficulty in power amplifiers. Therefore, such approach is not feasible for practical applications.

\section{MULTIMEDIA TRANSMISSION VIA VBLAST}

Multimedia data is first decomposed into layers with different perceptual importance. The channel performance can be measured as the average mean square error(MSE). It can be computed as $D_{c}=\sum_{l=0}^{N_{,}-1} W_{l} P e_{l}$, where $P e_{1}$ represents the error performance of source layer $l$ and $W_{l}$ represents the importance measure or QoS measure of layer l. The corresponding VBLAST optimization is to distribute the source layers to the appropriate channel layers to minimize the overall MSE. That is

$$
\begin{aligned}
\min & D_{c}=\sum_{k=0}^{N_{s}-1} W_{k} P e\left(E^{k}\right) \\
\text { subject to } & \sum_{k=0}^{N_{s}-1} S_{k} E^{k}=E_{b u d g e t},
\end{aligned}
$$

Here we assume the number of source layers $N_{s}$ is equivalent to the number of transmit antennas $N$. In addition, the source layers are of the same bit length, $S_{k}=S$. This design is still applicable to source layers of different sizes, simply by further dividing each source layer into sub-layers of the same size.

\subsection{Optimization for Serial Transmission}

Multimedia is a mixture of multiple concurrent services, such as video, voice over IP(VOIP) and data. Here, each service corresponds to a source layer. By applying serial transmission, the source layers are time multiplexed and during each transmission, only data stream from one service is transmitted. Figure 2 demonstrates the system architecture of serial transmission. The optimization is to allocate the transmitted power to each service in order to minimize the error performance. As explained in previous section, this is equivalent to

$$
\begin{aligned}
\min & D_{c}=\sum_{k=0}^{N_{a}-1} W_{k} P e_{a v g}\left(E_{T}^{k}\right) \\
\text { subject to } & \sum_{k=0}^{N_{s}-1} S_{k} E_{T}^{k}=E_{\text {budget }},
\end{aligned}
$$




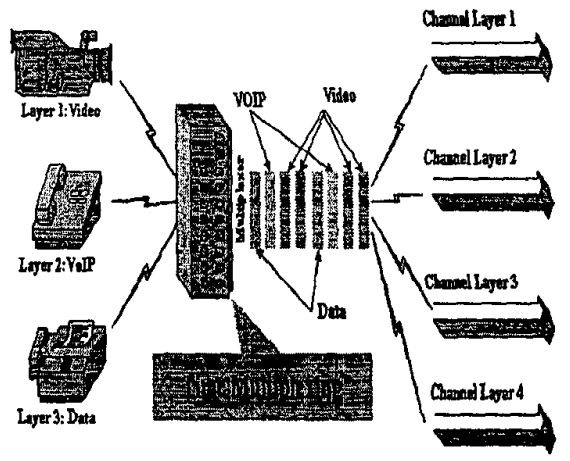

Figure 2: Serial Transmission in VBLAST. This example assumes 4 transmitter antennas(channel layers); 3 source layers, e.g. video, VoIP and data. The source layers are time multiplexed where video is assigned with two time slots, and VoIP and data are assigned with one each.

where $N_{s}$ is the number of source layers, $S_{k}=S$ is the symbol length of layer $k, P e_{\text {avg }}$ is the SER performance using equal power among the antennas. Similar to previous sections, LaGrange multiplier method can be applied to find the optimal solution.

\subsection{Optimization for Parallel Transmission}

For VBLAST, channel layers are associated with different error performance. Rather than equalizing or averaging the error performance among all the channel layers, we propose to transmit different source layers as different channel layers, as shown in Figure 3. We assume $N=N_{s}$ so that each source layer is mapped to one channel layer. It should be noted that the system can arrange the source layers into sublayers to support such assumption. This system design is to provide parallel transmission in space domain. The corresponding optimization is to minimize the overall error performance, through the appropriate power and possible coding rate allocation. Mathematically,

$$
\begin{array}{cl}
\min & \sum_{k=0}^{N s-1} W_{k}\left(\frac{E_{k} d^{2}}{4 N_{0}}\right)^{-T_{k}(M-k)} \\
\text { subject to } & \sum_{k=0}^{N s-1} S_{k} E_{k}=E_{\text {budget }} .
\end{array}
$$

Here we assume that $S_{k}=S, k=0 \ldots N-1$. Using LaGrange multiplier, the optimal power distribution satisfies

$$
E_{k}=\frac{4 N_{0}}{d^{2}}\left(\frac{W_{k} T(M-k)}{S \lambda}\right)^{\frac{1\left(M^{2}-k\right)+1}{2}}
$$

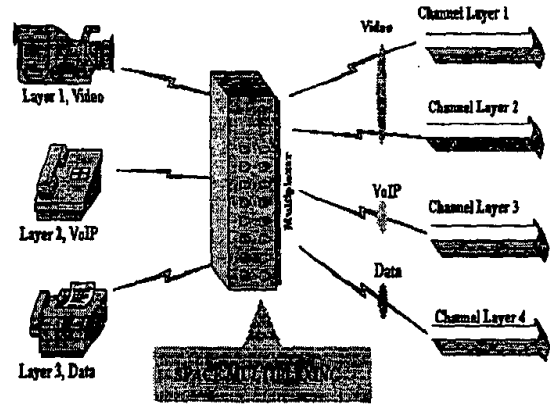

Figure 3: Parallel Transmission in VBLAST. The source layers are space multiplexed where they occupy different antennas. Video service occupies two antennas while the others occupy one each.

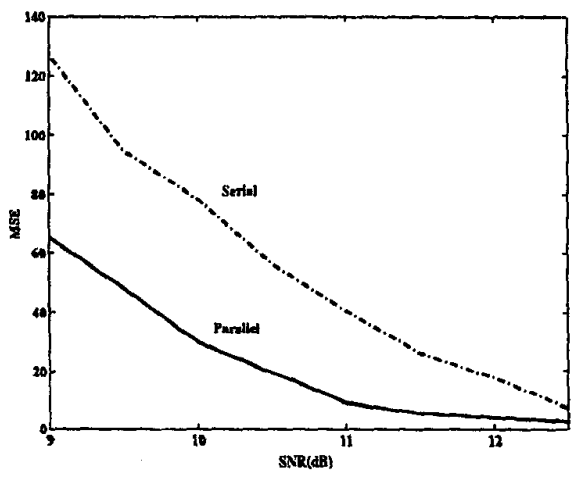

Figure 4: MSE Performance Comparison of serial and Parallel Transmission

and $\lambda$ satisfies

$$
\sum_{k=0}^{N-1} S \frac{4 N_{0}}{d^{2}}\left(\frac{W_{k} T(M-k)}{S \lambda}\right)^{\frac{1}{T(M-k)+1}}=E_{b u d g e t} .
$$

Since the left hand side is a monotonic function of $\lambda$, the optimal solution can be computed using Newton or bisection methods.

\section{SIMULATION RESULT}

The transmission system employs 7 transmit antennas and 7 receive antennas. We apply Reed Solomon $(7,3)$ code with 8PSK modulation. Therefore, each diagonal contains 7 8PSK symbols or a RS block. The corresponding parameters are $d^{2}=0.54$ and $T=5$. For simplicity, we assume the source multimedia data are decomposed into 7 equal length source layers, each associated with a weighting factor $W_{k}, k=0, \ldots, N-1$. For serial transmission, we apply equal power distribution described in (2). We compare serial and parallel 


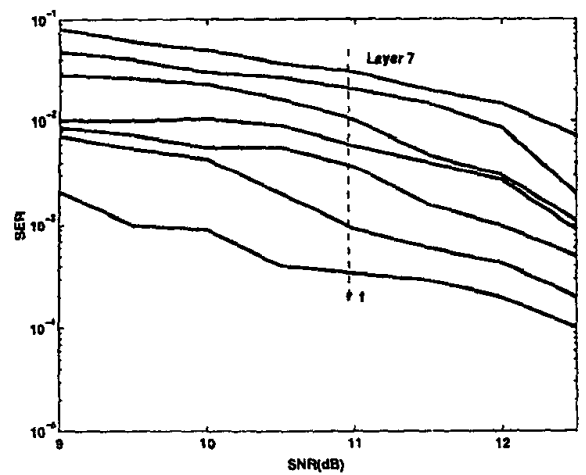

Figure 5: Serial Transmission: Layer Symbol Error Rate Distribution

transmission in terms of the mean square error(MSE) of the transmitted and received data. As shown in Figure 4, parallel transmission outperforms serial transmission, especially in low SNR region. As SNR increases, the performances converge.

We are interested in analyzing the error performance at each layer. Figure 5 and 6 demonstrate the symbol error rates(SER) at different layers for serial and parallel transmissions, respectively. We observe that major differences are located at layer 1,2 and 3, which correspond to the most important layers. They are performance bottleneck for serial transmission and to support which, large amount of transmitted power is consumed to reduce the noise effect at channel layers $N-1, N-2 \ldots$

\section{CONCLUSION}

By matching the source layers to channel layers in Layered space-time architecture, we propose a parallel transmission scheme utilizing the natural unequal error protection(UEP) in VBLAST. Power allocation is performed to optimize the error performance according to the importance of the source layers. However, the space-time codes rely on being able to accurately estimate the fading coefficients which are assumed to vary slowly. Therefore, powerful and yet efficient channel estimation or equalization techniques is very essential. Future work may include incorporating the channel estimation into the system and investigating the overall performance, especially the sensitivity to estimation error.

\section{REFERENCES}

[1] G. W. Wornell and M.D. Troot, "Efficient Signal Processing Techniques for Exploiting Transmit Antenna Di-

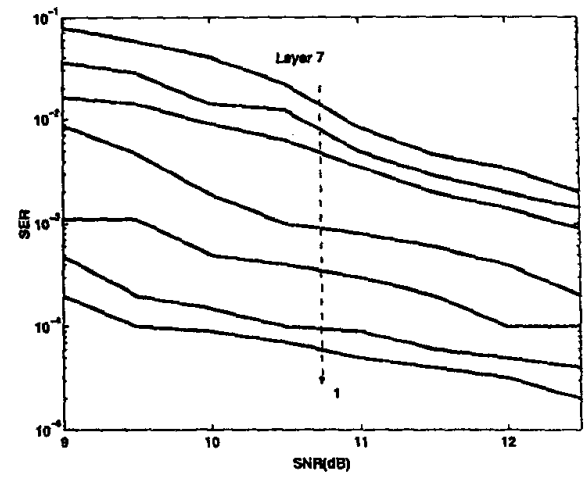

Figure 6: Parallel Transmission:Layer Symbol Error Rate Distribution

versity on Fading Channels", IEEE Trans. on Signal Processing, pp. 191-205, Jan. 1997.

[2] J.H.Winters, "The Diversity Gain of Transmit Diversity in Wireless Systems with Rayleigh Fading", Proc. of ICC'94, pp.1121-1125, June 1994.

[3] G. J. Foschini and M. J. Gans, "On Limits of Wireless Communications in a Fading Environment When Using Multiple Antennas", Wireless Personal Communications, pp. 311, Vol. 6, No. 3, March 1998.

[4] G. J. Foschini, "Layered Space-Time Architecture for Wireless Communication in a Fading Environment When Using Multiple Antennas" , Bell Labs Technical Journal, pp 41-59, Vol. 1, No. 2, Autumn 1996.

[5] G. D. Golden, G. J. Foschini, R. A. Valenzuela, P. W. Wolniansky, "Detection Algorithm and Initial Laboratory Results using the V-BLAST Space-Time Communication Architecture", Electronics Letters, Vol. 35, No. 1, Jan. 1999, pp. 14-15.

[6] D. Shiu and J.M. Kahn, "Design of High-Throughput Codes for Multiple-Antenna Wireless Systems", submitted to IEEE Trans. on Information Theory, Jan. 1999.

[7] V. Tarokh, N. Seshadri and A.R. Calderbank, "SpaceTime Codes for High Data Rate Wireless Communication:Performance analysis and code construction", IEEE Trans. on Information Theory, pp.744-765, vol.44, No,2, March 1998.

[8] H. Zheng and K.J.R. Liu, "Robust Image and Video Transmission over Spectrally Shaped Channels Using Multicarrier Modulation", IEEE Trans. on Multimedia, pp. 88-103, Vol.1, No.1 March, 1999. 